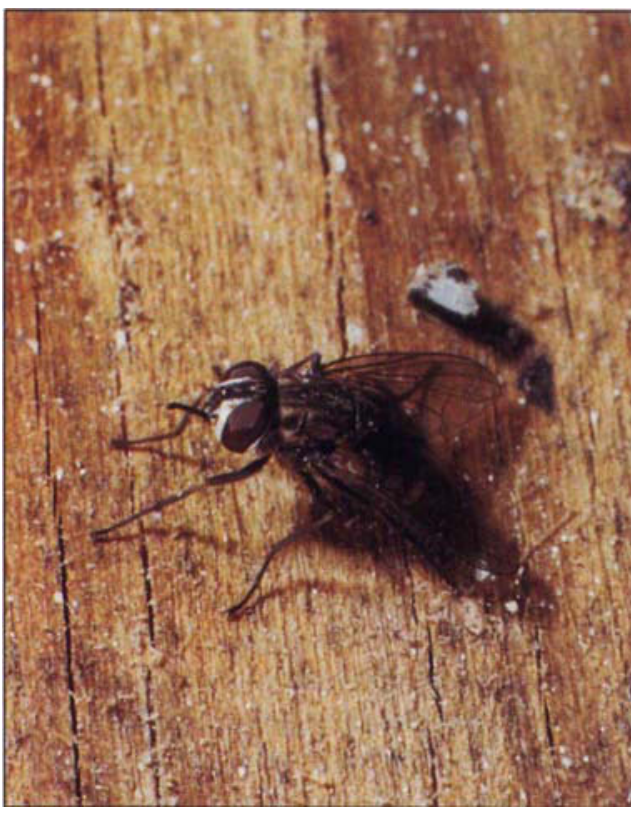

Biting stable flies rest on surfaces with their bodies at an angle and their bayonetlike mouthparts extending forward. The mouthparts have rasping teeth at the tip to lacerate the skin during blood feeding.

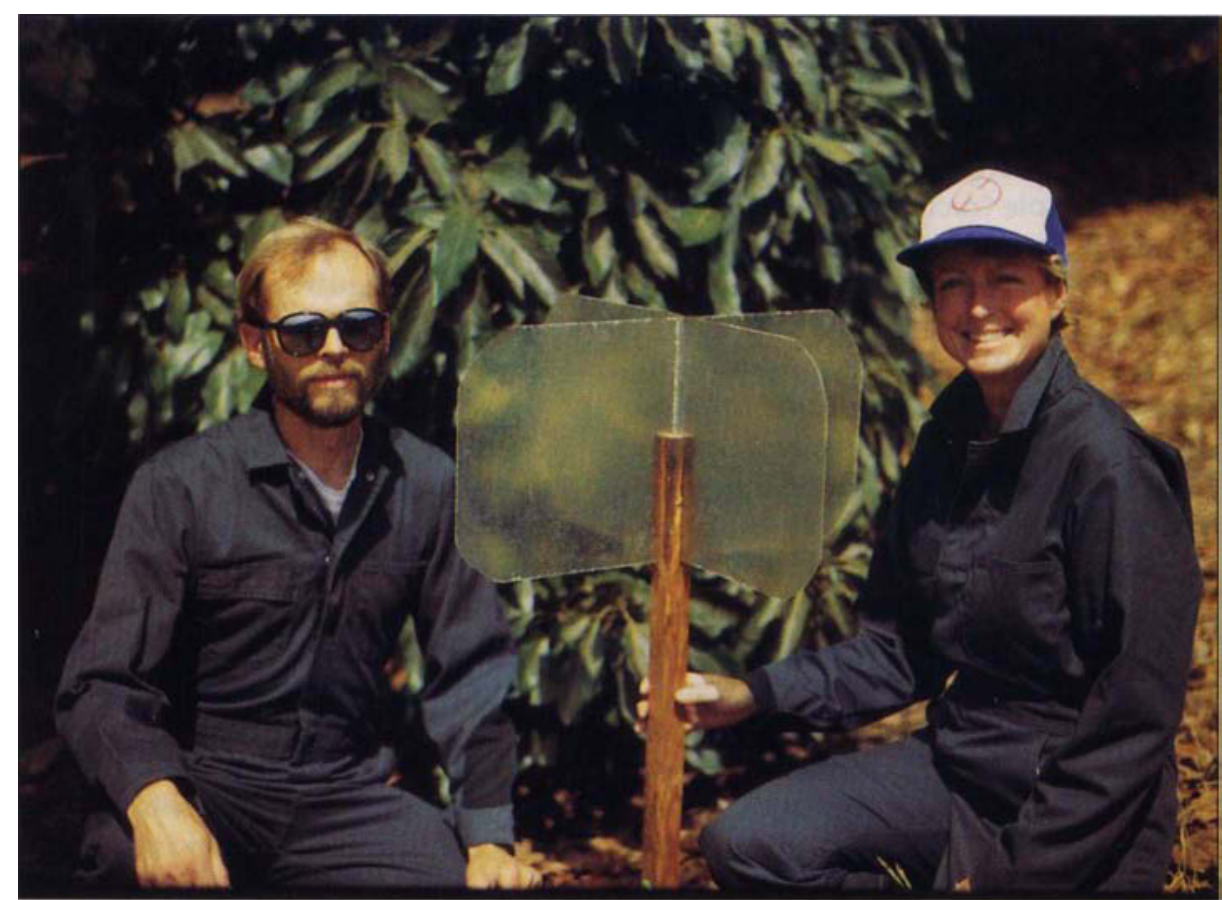

Alsynite fiberglass traps covered with adhesive are useful in monitoring stable fly numbers at dairies.

\title{
Octenol fails to lure stable fly to insecticide
}

\author{
Bradley A. Mullens $\square$ Nyles Peterson $\square$ Coralie E. Dada $\square$ Robert K. Velten
}

\section{A study was designed to determine whether blue targets were attractive to stable flies, whether octenol, a natural component of cow breath, enhanced attraction, and whether treating these targets with insecticide might enhance stable fly control. Blue cylindrical cloth targets treated with insecticides attracted resting stable flies in Southern California dairies. Octenol did not increase this attraction to the targets. Targets did not result in fewer flies in treated dairies, possibly due to insecticide resistance in these flies and the relatively small proportion of flies attracted.}

Dairy production is the top agricultural commodity in California, with an annual value of approximately $\$ 2$ billion. Actual value to the state's agricultural economy is much higher, given the accompanying demands such as alfalfa hay. Although a number of pasture-type operations still exist in California, particularly in the northern part of the state, most of the milk is produced on much larger, confinement-type operations in the Central Valley and southern counties. These large dairies are efficient, but they also result in large accumulations of feedstuffs and manure in very restricted areas. Such organic materials, particularly when handled improperly, can produce huge numbers of muscoid flies.

The primary species of concern are the common house fly, Musca domestica, and the biting stable fly,
Stomoxys calcitrans. The house fly is a nonbiting, nuisance pest, primarily a problem to residents on and near the dairies, but is not known to affect the cattle or milk yields adversely. The stable fly, on the other hand, is a severe biting pest of cattle that sometimes bites people as well.

Initial UC studies in Central and Southern California demonstrated that adult stable flies are present yearround, but have a very distinct spring (April to June) peak in abundance. This is probably a result of winter and spring conditions, particularly rainfall, that favor stable fly development in moist organic materials. Both sexes feed on blood, attacking mainly the legs and lower body regions. Unlike mosquitoes, female stable flies must feed several times to develop each egg batch. The impact of stable flies on milk yield and quality can be consider- 
able, particularly where numbers are high.

Reducing stable fly numbers can be difficult. Stable fly developmental sites in California dairies include spilled feed near feed aprons and commodity pits, old manure accumulations under fencelines and water troughs and other similar areas. Cultural control (sanitation) thus is important. Naturally occurring biological control by beetle and mite predators and pteromalid pupal parasites (small wasps) is very significant as well. Direct insecticide applications to lactating cattle are basically limited to pyrethrins or pyrethroids; washing the milking cows two or three times a day, a necessary part of the milking procedure, tends to remove insecticide residues.

Studies of adult stable fly activity have confirmed the value of adhesive-coated alsynite fiberglass traps for assessing relative adult fly abundance, and the correlation between adult collections on these traps and biting intensity on cattle is high. Trapping technology has improved markedly over the past several years. It has been known for some time that alsynite fiberglass reflects ultraviolet wavelengths that are attractive to stable flies, and traps made from this material have been widely used for sampling. The smooth, hard surface is poorly suited to pesticide applications; nevertheless, at least two studies in other states have shown local reduction of stable flies with insecticidetreated alsynite traps. Tsetse flies in Africa are attracted to pesticidetreated targets of blue fabric, baited with cheap, stable attractants such as cattle urine and 1-octen-3-ol (octenol), a natural component of cow breath. Such traps can reduce tsetse fly populations. Recent African studies also showed that blue-dyed fabric attracted three times as many stable flies as did

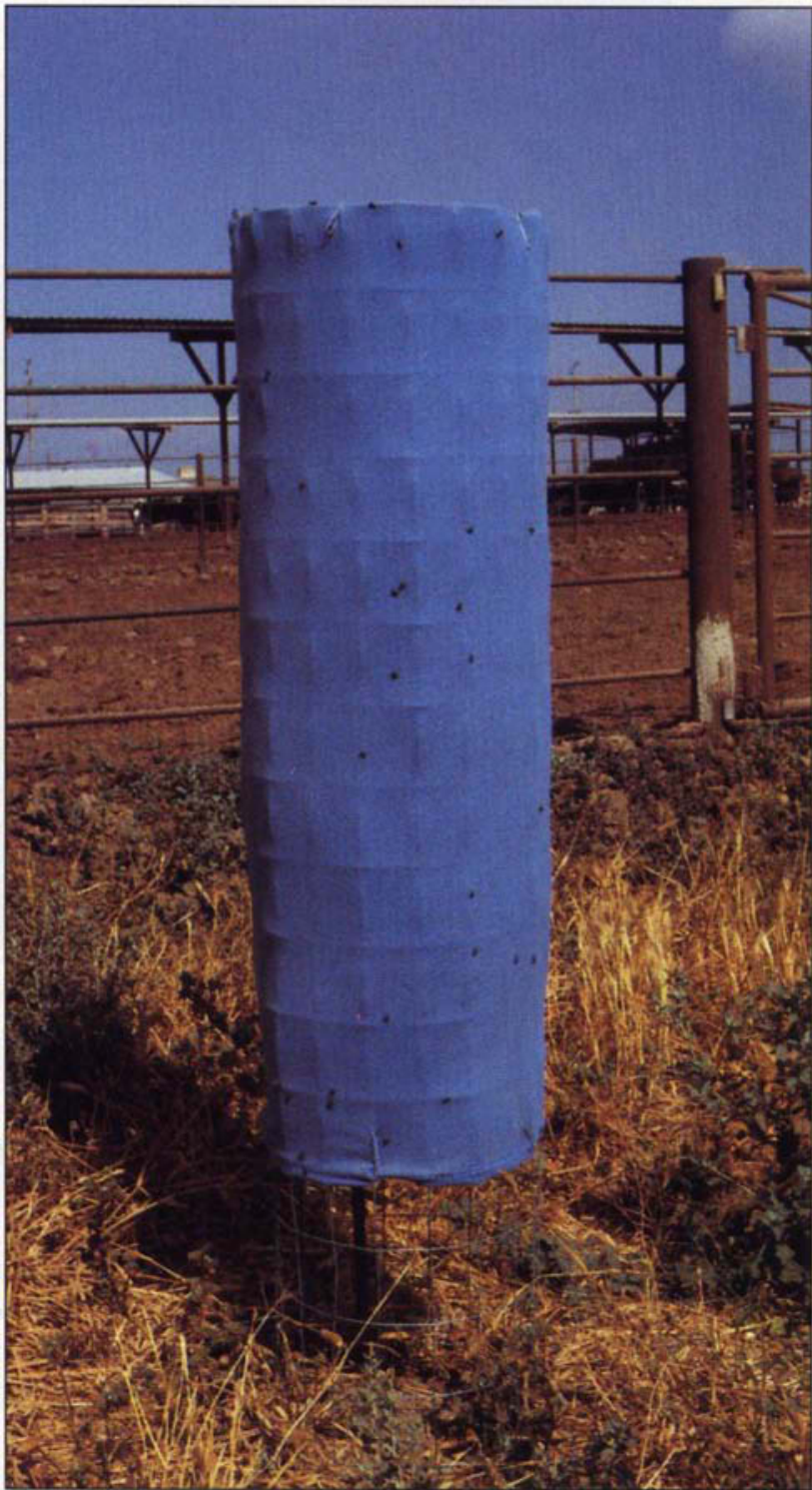

four sticky (Tack-Trap) alsynite fiberglass panel traps were deployed at each of the dairies; flies were counted and traps recoated weekly. Biting intensity on the cows was assessed weekly using fly counts on the outside of one front leg and the inside of the other on each of five randomly chosen cows in the vicinity of each trap (that is, 20 cows per dairy on each date). On May 14 two of the dairies (J, LM) were selected to receive the experimental targets.

The targets were wire frame cylinders, 4 feet tall and 1 foot in diameter; the top 3 feet of each target was covered with bright blue cloth. Targets were held in place by wiring them to pieces of $1 / 2$-inch-diameter reinforcing rod driven into the ground. Twenty targets were deployed in pairs, 20 feet apart, in each of 10 locations at each treated dairy. One target from each pair was randomly selected to receive a small capillary tube $(0.37-\mathrm{ml}$ volume $)$ of octenol containing a pipecleaner wick. The wick extended from the bottom of the tube to the top edge ( 3 inches). Release rates from these wicks

Experimental blue cylinder targets attract resting stable flies.

alsynite fiberglass traps; the addition of octenol nearly doubled the number of flies caught. The present study was designed to determine whether blue targets were attractive to stable flies, whether octenol enhanced attraction, and whether treating these targets with insecticide might enhance stable fly control.

\section{Flies counted, biting assessed}

Four confinement dairies in the Chino Basin of Riverside and San Bernardino counties were selected for study and designated J, CA, C and LM. Stable flies were monitored in two ways. Beginning in early April 1993, were calibrated to be approximately $0.6 \mathrm{mg} / \mathrm{hr}$, a rate that was attractive in earlier tests with Stomoxys in Africa. Vials were weighed the morning before deployment and 1 week later to determine actual release rates for that week. Vials were affixed to the top inside of the wire cylinder.

The outside surfaces of all targets (with and without octenol) were sprayed using a 3-gallon pressurized tank sprayer with $250 \mathrm{ml}$ (about $1 \mathrm{cup}$ ) of lambdacyhalothrin insecticide $(0.03 \%)$ per target. Two weeks later they were sprayed again with cyfluthrin insecticide $(0.1 \%)$. At each weekly visit resting stable flies on each target were visually counted from a distance of approximately 15 to 20 feet. The two targets in a pair were 
counted within 1 minute of each other from the same orientation. Flies were observed as well as collected directly from the targets, using a sweep net, to confirm fly species.

\section{Trap counts more consistent}

Stable fly numbers on the sticky traps and cow legs were low to moderate as the study began. Julian dates, which run from January 1 (Julian date $=1$ ) through December 31 (Julian date = 365), are used in Figure 1. Trap counts were more consistent than leg counts, because leg counts were more subject to weather conditions and other variables at the time of observation. Rainfall during the prior winter was well above normal, but only trace amounts of rain were recorded at the UC Riverside Experiment Station during April and May of 1993. Average temperatures during April, May and June were approximately $4^{\circ}, 3^{\circ}$ and $2{ }^{\circ} \mathrm{C}$ above normal, respectively.

The CA Dairy (untreated) had the highest numbers of flies initially and for the duration of the study (fig. 1). The blue targets were deployed as fly populations peaked in mid-May. $\mathrm{Nu}$ merous episodes of cattle bunching in the lots, a common behavioral response to biting fly pressure, were seen. No reduction of stable flies on the sticky traps or on the cows themselves was evident at dairies that received blue targets. The trap and leg counts were very high relative to earlier surveys in the same area during a drought period, confirming that wet winter weather tends to stimulate stable fly population growth the following spring. The general seasonal pattern, with a peak in fly populations in May, was consistent with earlier UC surveys in Southern and Central California.

By observation, flies on the blue targets or blue plastic barrels and tarps at the dairies were nearly always biting stable flies rather than house flies. Flies were netted from the blue targets 2 days after the cyfluthrin treatment at the J Dairy and from a fenceline at the nearby untreated CA Dairy on the same day. Of 23 flies from the blue targets, 22 were stable flies, despite the fact that house flies were becoming abundant by that time. At the CA
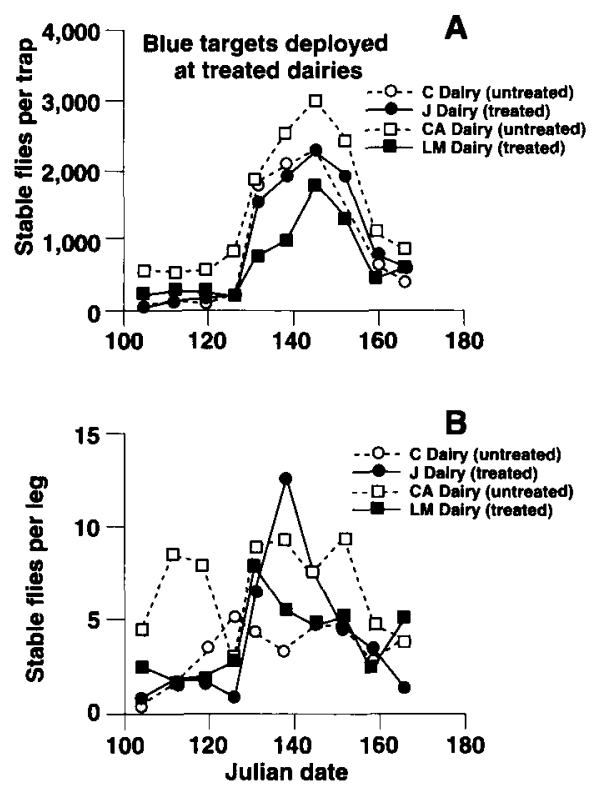

Fig. 1. Stable flies were most abundant in May at the four test dairies. Deployment of 20 insecticide-treated targets per dairy did not reduce stable fly numbers on fiberglass sticky traps (A) or on the cows (B). Solid lines and symbols are the treated dairies.

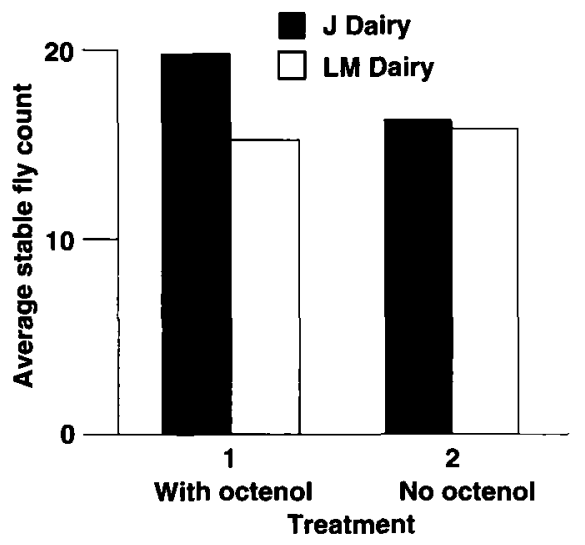

Fig. 2. Octenol, a natural component of cow breath, did not enhance stable fly attraction to the targets.

Dairy, 29 of 32 flies from the fenceline were stable flies. Collected flies were held in clean glass vials in the shade for 1 hour, after which $77 \%$ of flies from the blue targets were dead versus $22 \%$ of the control flies. The difference in mortality was highly significant ( $P$ $<0.01$ ) and indicated that contact with the targets resulted in mortality. Knockdown, however, was relatively slow, as dead flies were not seen around the base of the targets.

Actual octenol release rates were $0.56 \pm 0.03 \mathrm{mg} / \mathrm{hr}$ at J Dairy and $0.61 \pm$ $0.07 \mathrm{mg} / \mathrm{hr}$ at LM Dairy. Release rates were very close to the intended rate of $0.6 \mathrm{mg} / \mathrm{hr}$ and were not statistically different between dairies by paired $t-$ test $(P>0.5)$. Average numbers of flies resting on the targets varied substantially by date; at the peak of stable fly activity it was common to see 30 to 100 flies resting on one side of a target. However, numbers resting on octenolbaited versus unbaited targets at either dairy were not significantly different by a Mann-Whitney U-test $(P>0.05)$ (fig. 2).

Stable flies clearly detected octenol in prior laboratory electroantennogram experiments in England, but these studies did not assess whether the response was reflected behaviorally. Our results do not support the contention that $S$. calcitrans is attracted to octenol at this rate, as was shown in Africa. However, the African studies used targets that were remote from livestock, and it is quite possible that in the present studies effects of artificial host odors such as octenol were swamped out by the close proximity of large numbers of cattle.

\section{Targets ineffective}

In summary, blue surfaces do appear to be attractive for stable flies. However, it was not the intention of the present studies to investigate color attraction experimentally. Octenol does not appear to be a useful stable fly attractant, at least at the present release rates in the vicinity of cattle. The ineffectiveness of the targets and lack of knockdown observed also suggest insecticide resistance in the stable fly populations in the field, a possibility that should be investigated further. In some settings, such as pasture-type dairies with lower concentrations of animals, targets might still be useful, and this should be evaluated also.

B.A. Mullens is Professor and C.E. Dada and R.K. Velten are Staff Research Associates in the Department of Entomology, UC Riverside; N. Peterson is Dairy Farm Advisor, Cooperative Extension, San Bernardino County. We appreciate the financial support of the California Dairy Research Foundation and the dairymen who allowed us to use their dairies in the test. 